\title{
LA FERTIRRIGACIÓN CON VINAZA DE CAÑA DE AZÚCAR LIMITA LA TASA FOTOSINTÉTICA DE SOJA (GLYCINE MAX, LEGUMINOSAE)
}

\author{
SUgARCANE VINASSE FERTIRRIGATION NEGATIVELY AFFECTS LEAF \\ PHOTOSYNTHETIC RATES IN SOYBEAN (GLYCINE MAX, LEGUMINOSAE)
}

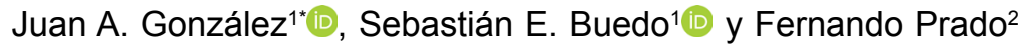

\begin{abstract}
1. Fundación Miguel Lillo Instituto de Ecología - Miguel Lillo 251 - (4000) Tucumán (Argentina). 2. Facultad de Cs. Naturales e IML, Universidad Nacional de Tucumán, Cátedra de Fisiología Vegetal, Miguel 205, (4000) Tucumán, Argentina.

*jagonzalez@lillo.org.ar

Citar este artículo GONZÁLEZ, J. A., S. E. BUEDO \& F. PRADO. 2019. La fertirrigación con vinaza de caña de azúcar limita la tasa fotosintética de soja (Glycine max, Leguminosae). Bol. Soc. Argent. Bot. 54: 215-223.
\end{abstract}

DOI: http://dx.doi. org/10.31055/1851.2372.v54. n2.24366

Recibido: 22 Noviembre 2018 Aceptado: 13 Marzo 2019 Publicado: 30 Junio 2019 Editor: Federico Mollard (D)

\section{SUMMARY}

Background and aims: A large volume of vinasse is generated annually in Argentinean ethanol producing provinces. It has been proposed that vinasses could be used for sugarcane or soybean fertigation. However, basic research to know the effect of vinasse on soil, morphology and crop physiology is scarce in the Argentine northwest.

M\&M: We provide data on the effect of diluted vinasse $(1: 1,1: 5$ and $1: 10 \mathrm{v} / \mathrm{v})$ on photosynthetic $\mathrm{CO}_{2}$ assimilation in soybean plants grown under semicontrolled conditions (greenhouse).

Results: Maximum photosynthetic assimilation rate $\left(A_{\max }\right)$ was strongly affected by all vinasse dilutions. In the lowest dilution $(1: 1)$ the $A_{\max }$ decreased $35 \%$ while in highest dilution (1:10) a $15.9 \%$ decrease was observed. Changes in $A_{\max }$ were correlated with decreases in stomatal conductance, leaf nitrogen content $\left(N_{f}\right)$ and total chlorophyll. Data of chlorophyll fluorescence show a decrease in $F_{v} / F_{m}$ at 1:1 dilution, which indicate a photosystem II (PSII) stress.

Conclusions: We can conclude that the application of diluted vinasse affects gas exchange parameters and if it is used it should be done with dilutions 1:10 or higher. Long-term studies are essential to know the effect of vinasse on physicochemical and biological characteristics of soils as well as the probable accumulation of heavy metals in fertirrigated plants.

\section{KEY WORDS}

Vinasse, contamination, soybean, stress, photosynthesis, fluorometry, phytoremediation, amendments.

\section{RESUMEN}

Introducción y Objetivos: En las provincias argentinas productoras de alcohol se genera anualmente un gran volumen de vinazas. Se ha propuesto que las mismas podrían ser utilizada para el fertirriego de la caña de azúcar u otras especies. Sin embargo, las investigaciones básicas para conocer el efecto de las mismas sobre el suelo, morfología y fisiología de las especies a fertirrigar son escasas en el noroeste argentino.

M\&M: Se aportan datos sobre el efecto de la vinaza diluida (1:1, 1:5 y 1:10 v/v) sobre la asimilación fotosintética de soja cultivada bajo condiciones semicontroladas (invernáculo).

Resultados: La asimilación fotosintética máxima $\left(A_{\max }\right)$ se afecta fuertemente en todas las diluciones utilizadas. En la menor dilución (1:1) la $A_{\max }$ disminuye el $35 \%$ respecto al control, mientras que en la dilución 1:10 dicho porcentaje se reduce al $15,9 \%$. Los cambios en la $A_{\max }$ se correlacionaron con disminuciones en la conductancia estomática, contenido de nitrógeno foliar $\left(\mathrm{N}_{\mathrm{f}}\right)$ y clorofila total. Los datos de fluorescencia de la clorofila muestran una disminución de $F_{v} / F_{m}$ en la dilución 1:1, lo que indicaría un estrés en el fotosistema II (PSII) inducido por la vinaza.

Conclusiones: Se concluye que la aplicación de vinaza diluida, afecta el intercambio de gases y que si se la utiliza debería hacerse con diluciones 1:10 o superiores. Se recomienda estudios a largo plazo para conocer el efecto de la vinaza sobre las características fisicoquímicas y biológicas de los suelos, así como la probable acumulación de metales pesados en las plantas fertirrigadas.

\section{Palabras clave}

Vinaza, contaminación, soja, estrés, fotosíntesis, fluorometría, fitoremediación, enmienda. 


\section{INTRODUCCIÓN}

El auge en la producción de bioetanol a partir de la caña de azúcar se ha visto potenciado por el uso creciente del mismo como combustible alternativo a los derivados del petróleo en la industria automotriz (Álvarez et al., 2008). Si bien este hecho ha permitido una cierta recuperación económica en la industria azucarera, no es menos cierto que ello también ha traído aparejado una serie de inconvenientes ambientales que de no mediar una pronta solución, puede convertirse en una problemática de magnitud impredecible. El proceso de producción de alcohol a partir de la fermentación de la melaza origina grandes volúmenes de un desecho altamente contaminante conocido con el nombre de vinaza (Larrahondo, 2009). Su producción es muy elevada ya que por cada litro de alcohol obtenido se generan aproximadamente 13 litros de vinaza (Chanfón Curbelo \& Lorenzo Acosta, 2014). La vinaza se caracteriza por presentar un $\mathrm{pH}$ bajo (entre 4,8 y 5,4 ), altos valores de DQO (demanda química de oxígeno) (entre 90.000 a $150000 \mathrm{mg} / \mathrm{L}$ ) y DBO (demanda biológica de oxígeno) (32.000 a 54.000 $\mathrm{mg} / \mathrm{L})$, elevado porcentaje de materia orgánica (10 $\%$ entre orgánica e inorgánica) y alto contenido de sólidos totales (promedio de $50.000 \mathrm{mg} / \mathrm{L}$ ), entre otros componentes (España-Gamboa et al., 2011). Por esta razón, se la considera como un residuo que impacta negativamente en el ambiente, especialmente el hídrico, ya que a los problemas antes mencionados debe sumarse que debido a su coloración marrón oscuro disminuye la luminosidad de las aguas y la actividad fotosintética de algas y plantas acuáticas sumergidas (Christofoletti et al., 2013), lo que conduce a la eutrofización de los cuerpos de agua. Aunque las vinazas provocan impactos negativos sobre el medio ambiente cuando no son tratadas o dispuestas adecuadamente; las mismas, sin embargo, pueden ser empleadas de diversas maneras ya sea, como energía renovable en la producción de biogás, para la alimentación animal en la producción de levadura o como sustituto de fertilizantes en la producción agrícola (Pérez-Bermúdez \& Garrido-Carralero, 2006). Esta última opción ha sido hasta el presente la más utilizada sobre todo en el cultivo de la caña de azúcar (Chanfón Curbelo \& Lorenzo Acosta, 2014). Trabajos de investigación realizados en diversos países, especialmente en Brasil (Sanchez Moore et al., 2017), revelan que la fertirrigación con vinaza incrementa la productividad de la caña de azúcar, evidenciándose con ello que bajo condiciones racionales de manejo puede sustituir parcial o totalmente la fertilización mineral (Muñoz, 2009). Si bien, en la región NOA de nuestro país, se llevan adelante proyectos de investigación vinculados al uso de la vinaza en el cultivo de la caña de azúcar (Morandini \& Quaia, 2013), los volúmenes producidos son extremadamente grandes por lo que resulta necesario explorar la utilización de la vinaza como fertilizante en otros cultivos agrícolas de carácter intensivo como soja, maíz, trigo y girasol, entre otros. Sin embargo, es necesario mencionar que existen datos sobre la presencia de varios metales, algunos pesados, que las vinazas contienen (como bario, cromo, cobre, mercurio, molibdeno, níquel y cinc, entre otros) (Christofoletti et al., 2013; Robertiello, 1982) que sería necesario evaluar.

En un estudio previo sobre crecimiento y productividad de soja (Glycine max), cultivada en invernáculo e irrigada con diferentes diluciones de vinaza, se demostró que los efectos de ésta dependían de la dilución utilizada y de la variable fisiológica considerada (González et al., 2018). Entre las variables analizadas se encontró que el AFE (área foliar específica), que es una variable fuertemente correlacionada con la morfología y fisiología foliar, resultaba afectada no solo por el agregado de vinaza al sustrato sino también por la dilución en que ésta se aplicaba. Este hecho permite suponer que el efecto de la vinaza va más allá del simple aporte de nutrientes (fertilización), como el nitrógeno, ya que también podría estar afectando procesos fundamentales de la planta como ser la actividad fotosintética. En este sentido, resulta interesante destacar, que los trabajos referidos a los efectos de la vinaza sobre la actividad fotosintética (intercambio de gases y fluorescencia de la clorofila) resultan muy escasos a nivel internacional e inexistentes a nivel de la región NOA. Los estudios de la actividad fotosintética revisten mucha importancia, ya que los mismos pueden brindar información sobre el estrés que puede causar un determinado efluente cuando es usado como fertilizante (Brack \& Frank, 1998; González Moreno et al., 2008). Así el objetivo de este trabajo fue analizar el efecto de distintas diluciones de vinaza sobre los parámetros fotosintéticos y fluorométricos de plantas de soja cultivadas bajo condiciones semicontroladas. 


\section{J. A. González et al. - Vinazas y fotosíntesis en soja}

\section{Materiales y Métodos}

Material vegetal, condiciones de crecimiento, vinaza utilizada y suelo utilizado.

El material vegetal utilizado se eligió en función de contar con un crecimiento los más homogéneo posible de manera que cualquier efecto por aplicación del efluente se tradujera rápidamente en un cambio visible. Para ello se eligieron semillas certificadas de soja correspondientes a la variedad A-6126 (semillero NIDERA), cultivada en la región NOA. Las condiciones de crecimiento, el suelo y vinaza utilizados, así como los tratamientos a que fueron sometidas las plantas fueron similares a las descritas previamente (González et al., 2018).

\section{Intercambio de gases}

Las mediciones del intercambio de gases se realizaron con un equipo IRGA portátil (LICOR 6400 -XT, USA) equipado con una cámara fluorométrica. Las mediciones se realizaron entre las 10:00 y las 13:00 h en plantas de 75 - 80 días de desarrollo y tamaño similar, regadas a saturación. Por tratamiento se seleccionaron 3-4 plantas y en cada una de ellas se realizaron mediciones por triplicado o cuadriplicado a fin de obtener lecturas estables o con poca variación. Para estudiar el efecto de la densidad de flujo fotosintético de fotones (DFFF) sobre la respuesta fotosintética (curvas $\mathrm{A}_{\mathrm{n}} / \mathrm{DFFF}$ ), se utilizaron diferentes niveles de DFFF entre 2000 y $0 \mu \mathrm{mol} \mathrm{m} \mathrm{m}^{-2} \mathrm{~s}^{-1}(2000,1500$, $1000,500,400,300,200,100,50$ y 0). Los distintos niveles de DFFF fueron provistos por luz artificial (90\% de rojo y $10 \%$ azul) a partir de una fuente LED (LICOR 6400-02B), incorporada a la cámara fluorométrica. Se utilizó la cámara mencionada ya que con la misma se puede medir la fluorescencia modulada. La asimilación neta de $\mathrm{CO}_{2}\left(\mathrm{~A}_{\text {net }}\right)$, la transpiración foliar ( $\mathrm{Tr}$ ) y la conductancia estomática (CE) fueron medidas bajo las siguientes condiciones: intensidad lumínica $1.500 \mu \mathrm{mol} \mathrm{m}{ }^{-2}$ $\mathrm{s}^{-1}$, concentración de $\mathrm{CO}_{2} 400 \mathrm{ppm}$, temperatura foliar $\left(25 \pm 0,5^{\circ} \mathrm{C}\right)$ y humedad relativa entre 50 y 60 $\%$. La capacidad de carboxilación (CC) se obtuvo como el cociente entre $\mathrm{A}_{\text {net }}$ y $\mathrm{C}_{\mathrm{i}}$ (concentración interna de $\mathrm{CO}_{2}$ ); mientras que la eficiencia en el uso del agua (EUAi) se determinó a partir de la relación entre $A_{\text {net }} / C E$ y no a partir del cociente $A_{\text {net }} / T_{r}$ para eliminar el efecto de la temperatura y el gradiente de humedad sobre la transpiración (Rawson et al.,
1977). La fotosíntesis neta máxima $\left(\mathrm{A}_{\max }\right)$, el punto de saturación lumínica (PSL) (definido como la DFFF a la cual $A_{\text {net }}$ es igual al $90 \%$ de la $A_{\text {max }}$ ), el punto de compensación lumínica (PCL) (que corresponde al valor de DFFF en el cual $A_{\text {net }}$ es igual a cero), la respiración nocturna $\left(\mathrm{R}_{\mathrm{n}}\right)$ (definida como la $\mathrm{A}_{\text {net }}$ a un valor de $\mathrm{DFFF}=0$ ) y la eficiencia cuántica de la fotosíntesis $\left(\varnothing_{\mathrm{CO} 2}\right)$ (definida como el número de moles de fotones necesarios para fijar un mol de $\mathrm{CO}_{2}$ ) fueron calculadas de acuerdo al procedimiento de Schulte et al. (2003). La eficiencia del fotosistema II (ФPSII) se estableció a partir de la fluorescencia de la clorofila y que es calculada por el equipo utilizado. La Tasa de Transporte de Electrones (TTE), que representa el flujo de fotones en el fotosistema II, fue calculado a como:

$$
\mathrm{TTE}=\Phi \text { PSII } * \text { DFFF } * 0,5 * 0,84
$$

Las constantes 0,5 y 0,84 indican la fracción de energía de excitación distribuida al PSII para las plantas C3 y la fracción de luz incidente que es absorbida por las hojas respectivamente (Schreiber et al., 2000)

Las mediciones incluyeron también el cálculo de la fluorescencia de la clorofila $\left(\mathrm{F}_{\mathrm{v}} / \mathrm{F}_{\mathrm{m}}\right)$ que indica la eficiencia cuántica máxima del PSII y fue obtenida como:

$$
\mathrm{F}_{\mathrm{v}} / \mathrm{F}_{\mathrm{m}}=\left(\mathrm{F}_{\mathrm{m}}-\mathrm{F}_{\mathrm{o}}\right) / \mathrm{F}_{\mathrm{m}}
$$

Donde $\mathrm{F}_{\mathrm{o}}$ es la fluorescencia mínima (corresponde a la hoja adaptada a oscuridad), mientras que $\mathrm{F}_{\mathrm{m}}$ corresponde a la fluorescencia máxima que se alcanza después de un pulso saturante de luz (Maxwell \& Johnson, 2000). Las mediciones se realizaron en hojas aclimatadas a la oscuridad durante $30-40$ minutos.

\section{Análisis estadísticos}

Los resultados obtenidos se analizaron mediante el test de Shapiro para comprobar la normalidad de los datos y luego una prueba $F$ (Fisher) para determinar la homogeneidad de las varianzas. Para determinar la existencia de diferencias significativas entre los tratamientos $(p \leq 0,05$ y $p \leq$ 0,01 ), se aplicó el análisis de la varianza (ANOVA) utilizando el paquete estadístico Infostat (versión 1.1) (Universidad Nacional de Córdoba). 


\section{Resultados}

Respuesta de la asimilación fotosintética neta $\left(A_{n e}\right)$ a la densidad de flujo fotosintético de fotones (DFFF)

A valores bajos de DFFF $\left(0-500 \mu \mathrm{mol} \mathrm{m} \mathrm{m}^{-2}\right.$ $\mathrm{s}^{-1}$ ) no se observan diferencias significativas en los valores de $\mathrm{A}_{\text {net }}$ entre el control sin vinaza y los tratamientos con distintas diluciones de la misma (Fig. 1). A partir de los $600 \mu \mathrm{mol} \mathrm{m}^{-2} \mathrm{~s}^{-1} \mathrm{se}$ observan diferencias significativas entre el control y los tratamientos 1:1 y 1:5. Curiosamente con la mayor dilución de vinaza (1:10) se encontraron valores $\mathrm{A}_{\text {net }}$ menores al control, pero las diferencias no fueron significativas $(\mathrm{p} \leq 0,05)$. De este modo puede concluirse que el riego con vinaza afecta la $\mathrm{A}_{\text {net }}$ según cual sea la dilución aplicada. El valor de PSL en los tratamientos 1:1 y 1:5 mostró diferencias significativas $(\mathrm{p} \leq 0,05)$ respecto al control; mientras que a la dilución 1:10 tales diferencias no fueron detectadas. Por su parte, el valor de PCL practicamente no resultó afectado en ningún tratamiento, salvo en la dilución 1:10 (Tabla 1). La eficiencia cuántica de la fotosíntesis $\left(\varnothing_{\mathrm{CO} 2}\right)$ no presentó diferencias entre los diferentes tratamientos. En cuanto a la $\mathrm{R}_{\mathrm{n}}$ los valores medidos se incrementan con respecto al control, pero no resultan significativamente diferentes entre ellos. La TTE resultó afectada significativamente en las diluciones
1:1 y 1:5; mientras que a la dilución 1:10 tiende a recuperar su valor acercándose al control (Tabla 1). La $\mathrm{A}_{\max }$ disminuyó por el agregado de vinaza con valores que oscilaron entre un 15,9\% para la dilución 1:10 y $39 \%$ para la dilución 1:5, respectivamente.

Respuesta de la CE, Ci, T, EUA $A_{i}$ y CC a los tratamientnos con vinaza

Con la dilución 1:1 se observa una disminución significativa en los valores de $\mathrm{CE}, \mathrm{Ci}, \mathrm{T}_{\mathrm{r},} \mathrm{EUA}_{\mathrm{i}} \mathrm{y}$ CC (Tabla 2). Al aumentar la dilución (tratamientos 1:5) los valores de las variables mencionadas se incrementan pero luego en 1:10 disminuyen hasta aproximarse al control.

Correlaciones entre el contenido de clorofila total $\left(\mathrm{Cl}_{\text {tot }}\right)$, asimilación fotosintética máxima $\left(\mathrm{A}_{\max }\right)$ y contenido de nitrógeno foliar $\left(\mathrm{N}_{\mathrm{f}}\right)$.

Los datos de $\mathrm{N}_{\mathrm{f}}, \mathrm{Cl}_{\text {tot }}$ informado por González et al., (2018) se correlacionaron con la $\mathrm{A}_{\max }$ obtenida en este estudio. El mayor efecto sobre el contenido de $\mathrm{N}_{\mathrm{f}}$ se registra en la dilución 1:5 con la consecuente disminución en el contenido de $\mathrm{Cl}_{\text {tot }}$ $\mathrm{y}$ en el valor de $\mathrm{A}_{\max }$. Por otra parte, los valores de $\mathrm{Cl}_{\text {tot }}$ y $\mathrm{A}_{\text {max }}$ mostraron una alta correlación positiva con los valores de $\mathrm{N}_{\mathrm{f}}\left(\mathrm{Cl}_{\text {tot }}=0,8039 * \mathrm{~N}_{\mathrm{f}}+3,1033, \mathrm{R}^{2}\right.$ $=0,74$ y $\left._{\text {max }}=12,747 * \mathrm{~N}_{\mathrm{f}}-12,162, \mathrm{R}^{2}=0,96\right)$. Las reducciones en los parámetros mencionados tienden a disminuir a medida que la dilución se hace mayor para aproximarse a los valores del tratamiento

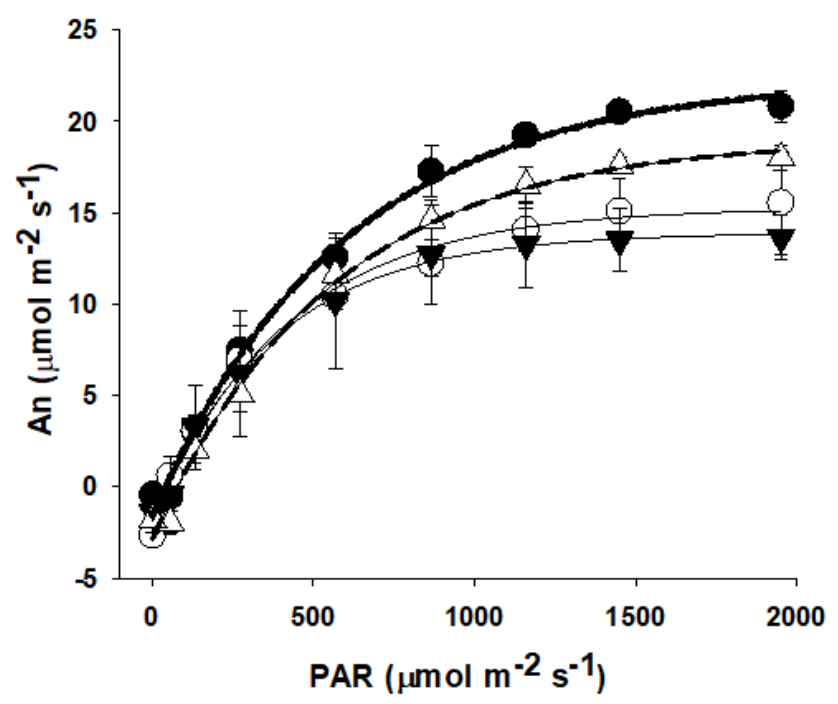

Fig. 1. Efecto del riego con vinazas diluidas sobre la Asimilación Neta del $\mathrm{CO}_{2}\left(\mathrm{~A}_{\mathrm{net}}\right)$ en condiciones de DFFF creciente. Cada medición es el promedio de $4-5$ plantas distintas. (•):Control; $(\circ)$ : $1: 1 ;(\boldsymbol{\nabla}): 1: 5$ y $(\Delta)$ 1:10 


\section{J. A. González et al. - Vinazas y fotosíntesis en soja}

Tabla 1. Asimilación fotosintética máxima $\left(A_{\max }\right)$, punto de saturación por luz (PSL), punto de compensación por luz (PCL), eficiencia fotosintética $\left(\varnothing_{\mathrm{CO} 2}\right)$, respiración nocturna $(\mathrm{Rn})$ y tasa de transporte de electrones (TTE) bajo distintas condiciones de riego con vinazas. Todos los valores consignados fueron obtenidos bajo luz saturante y corresponden al promedio de 5 plantas distintas. El valor entre paréntesis indica la DS. Letras minúsculas diferentes indican diferencias significativas $(p \leq 0,05)$.

\begin{tabular}{|c|c|c|c|c|c|c|}
\hline & $\begin{array}{c}A_{\max } \\
\left(\mu \mathrm{mol} \mathrm{m}^{-2} \mathrm{~s}^{-1}\right)\end{array}$ & $\begin{array}{c}\text { PSL } \\
\left(\mu \mathrm{mol} \mathrm{m}^{-2} \mathrm{~s}^{-1}\right)\end{array}$ & $\begin{array}{c}\mathrm{PCL} \\
\left(\mu \mathrm{mol} \mathrm{m}^{-2} \mathrm{~s}^{-1}\right)\end{array}$ & $\begin{array}{c}\varnothing_{\mathrm{co} 2} \\
\left(\mathrm{~mol} \mathrm{~mol}^{-1}\right)\end{array}$ & $\begin{array}{c}\mathrm{Rn} \\
\left(\mu \mathrm{mol} \mathrm{m}^{-2} \mathrm{~s}^{-1}\right)\end{array}$ & $\begin{array}{c}\text { TTE }_{\max } \\
\left(\mu \mathrm{mol} \mathrm{m}^{-2} \mathrm{~s}^{-1}\right)\end{array}$ \\
\hline Control & $22.6(1.6) \mathrm{a}$ & $1418.0(31.6) \mathrm{a}$ & $41.2(5.1) a$ & $0.039(0.01) a$ & $1.6(0.1) a$ & $98.76(6.1) \mathrm{a}$ \\
\hline $1: 1$ & $14.7(1.4) b$ & 1242.9 (53.3) b & $43(5.3) a$ & $0.035(0.01) a$ & $1.7(0.2) a$ & $50.66(2.1) b$ \\
\hline $1: 5$ & $13.6(0.5) b$ & $851.9(19.6) \mathrm{c}$ & 47.7 (2.9) a & $0.040(0.01) a$ & $1.9(0.1) a$ & $78.16(4.5) b$ \\
\hline $1: 10$ & $19.0(0.5) b$ & $1386.9(114.2) a$ & $53.6(2.3) \mathrm{b}$ & $0.034(0.02) a$ & $1.8(0.3) \mathrm{a}$ & $94.43(3.4) \mathrm{a}$ \\
\hline
\end{tabular}

Tabla 2. Efecto de las vinazas sobre la conductancia estomática (CE), concentración interna de $\mathrm{CO}_{2}$ $\left(C_{i}\right)$, transpiración foliar $\left(T_{r}\right)$, eficiencia intrinseca en el uso del agua (EUA $A_{i}$ ) y capacidad de carboxilación (CC). Todos los valores consignados fueron obtenidos bajo luz saturante y corresponden al promedio de 5 plantas distintas. El valor entre paréntesis indica la DS. Letras minúsculas diferentes indican diferencias significativas $(p \leq 0,05)$.

\begin{tabular}{|c|c|c|c|c|c|}
\hline & 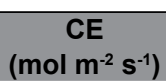 & $\begin{array}{c}\mathrm{C}_{\mathrm{i}} \\
\left(\mu \mathrm{mol} \mathrm{mol}^{-1}\right)\end{array}$ & $\begin{array}{c}\mathrm{T}_{\mathrm{r}} \\
\left(\mathrm{mmol} \mathrm{m}^{-2} \mathrm{~s}^{-1}\right)\end{array}$ & 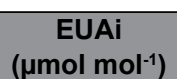 & 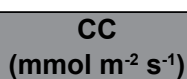 \\
\hline Control & $0.7(0.01) a$ & $76.1(12.0) \mathrm{a}$ & $2.52(0.49) \mathrm{a}$ & 19.65 (1.0) a & $213.8(4.5) a$ \\
\hline 1:1 & $0.3(0.01) b$ & 71.3 (18.5) a & $1.38(0.41) b$ & $16.27(1.5) b$ & $100.3(12.3) b$ \\
\hline $1: 5$ & $1.1(0.03) \mathrm{c}$ & $122.8(33.7) b$ & $3.94(0.86) c$ & $17.31(0.4) b$ & $156.1(9.4) \mathrm{c}$ \\
\hline $1: 10$ & $0.7(0.01) a$ & $97.1(19.7) \mathrm{a}$ & $2.89(0.44) a$ & $18.66(0.4) \mathrm{a}$ & $125.8(9.8) \mathrm{c}$ \\
\hline
\end{tabular}

control. Así por ejemplo en las diluciones 1:1 y 1:5 la $\mathrm{A}_{\max }$ disminuyó entre el 35 y $40 \%$, mientras que a la dilución 1:10 dicha reducción fue solo del $16 \%$.

Efecto de la vinaza sobre la fluorescencia foliar $\left(F_{v} y\right.$ $\left.F_{m}\right)$ y su relación con la integridad del fotosistema II (PSII)

La integridad del PSII, a través de mediciones de las variables $F_{v}$ (fluorescencia variable) y
$\mathrm{F}_{\mathrm{m}}$ (fluorescencia máxima) muestra que las diferentes diluciones de vinaza provocaron una disminución en los valores de $\mathrm{F}_{\mathrm{v}} / \mathrm{F}_{\mathrm{m}}$ (Fig. 2). Sin embargo, a medida que aumenta la dilución dicho valor se recupera hasta que en la dilución 1:10 prácticamente iguala al valor del control. El análisis estadístico demostró que solamente la dilución $1: 1$ afecta significativamente $(\mathrm{p} \leq 0,05)$ la relación $\mathrm{F}_{\mathrm{v}} / \mathrm{F}_{\mathrm{m}}$.

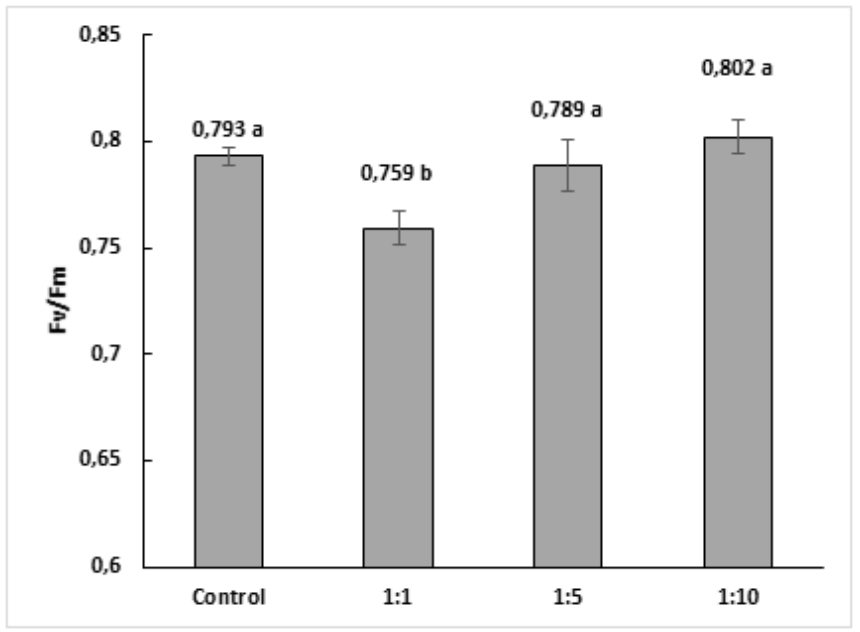

Fig. 2. Valores de eficiencia cuántica máxima del PSII (Fv/Fm) medidos en soja bajo distintos tratamientos con vinazas (diluciones 1:1, 1:5, 1:10). Cada valor es el promedio de $4-5$ plantas distintas y la barra vertical representa la DS. Letras iguales indican que no hay diferencias significativas $(p \leq 0,05)$ con respecto al control. 


\section{Discusión}

La aplicación de vinaza diluida afecta la $\mathrm{A}_{\max }$ en plantas de soja cultivadas en condiciones semicontroladas (invernadero). Dicho efecto resultó dependiente de la dilución utilizada, registrándose el mayor efecto con las diluciones 1:1 y 1:5 (valores diferentes en ambos casos, pero sin diferencia significativas entre ambas); mientras que con la mayor dilución (1:10) la $\mathrm{A}_{\max }$ tiende a aproximarse al valor control $\left(22,6 \mathrm{vs} 19 \mu \mathrm{mol} \mathrm{m}^{-2} \mathrm{~s}^{-1}\right)$. El menor valor de $A_{\max }$, registrado en el tratamiento 1:1 muestra una clara relación con el descenso en la CE. Así el cierre estomático parcial que ocurriría a la dilución 1:1 reduciría la difusión del $\mathrm{CO}_{2}$ al mesófilo, para luego recuperarse a medida que la dilución aumenta. En relación a la eficiencia cuántica de la fotosíntesis $\left(\varnothing_{\mathrm{CO} 2}\right)$ si bien los valores medidos no presentaron diferencias significativas entre los diferentes tratamientos, el valor más alto de $\varnothing_{\mathrm{CO} 2}$ fue de 0,039 para el control lo que resulta bajo para el valor medio de las especies C3 y que resulta ser de 0,052 (Skillman, 2008). Los valores medidos de $\mathrm{R}_{\mathrm{n}}$, en todas las diluciones no mostraron diferencias entre ellos, pero fueron ligeramente superiores al control. Sin embargo, los mismos resultaron mayores a los informados para especies que crecen a pleno sol (Björkman, 1968). Por otro lado, la TTE que es una factor importante a nivel del fotosistema II (PSII) es muy sensible al estrés hídrico (Salazar-Parra et al., 2012). Nuestros estudios sugieren que en las diluciones 1:1 y 1:5 el efecto por el estrés hídrico, impuesto por la salinidad de la vinaza, se traduce en una disminución de los parámetros medidos, en las diluciones mencionadas, y una recuperación hacia diluciones mayores (1:10 en este caso). En un trabajo previo (González et al., 2018) se demostró que ni la altura de la planta ni el diámetro del tallo resultaron afectados en todas las diluciones utilizadas sin embargo, el número de hojas sí se afectó en las diluciones 1:1 y 1:5. De esto surge que las diferencias observadas en la tasa de asimilación fotosintética en las diluciones 1:1 y 1:5 no tuvieron efecto sobre los parámetros antes mencionados y que los efectos producidos se habrían manifestado a nivel de otros órganos de la planta. En efecto, la masa foliar específica (MFE), que resulta ser un estimador de la inversión en carbono que la planta realiza para obtener una superficie determinada de hoja, si resultó afectada. En ese sentido, los resultados obtenidos demostraron que las hojas de las plantas regadas con las diluciones 1:1, 1:5 y 1:10 aumentaron su inversión en carbono en casi un 27 \% (González et al., 2018). Esto significa que las plantas regadas con vinaza aumentaron el espesor de lámina foliar, con el consiguiente incremento en la inversión de carbono para sostener dicha tendencia. Los valores de Tr y EUA (Tabla 2) que se registran en las diferentes diluciones disminuyen o aumentan su valor en concordancia con el cierre de las estomas (CE).

El nitrógeno es uno de los factores más importantes que afecta la fisiología de las plantas y limita el desarrollo y rendimiento de los cultivos (Milford et al., 1985; Vos \& Biemond, 1992; Trápani \& Hall, 1996; Gastal \& Lemaire, 2002; Marschner \& Marschner, 2012). En forma particular existe una estrecha correlación positiva entre el contenido de nitrógeno foliar $\left(\mathrm{N}_{\mathrm{f}}\right)$ y la actividad fotosintética (Field \& Mooney, 1986; Evans, 1989); lo que se explica por el hecho de que el nitrógeno forma parte de las enzimas del Ciclo de Calvin (Evans, 1989), tal el caso de la Ribulosa 1,5 Bisfosfato Carboxilasa Oxigenasa (RUBISCO) que representa el $50 \%$ de toda la proteína presente en la hoja (Lambers et al., 2008). En nuestros resultados se refleja claramente que la disminución del $\mathrm{N}_{\mathrm{f}}$ con las diluciones 1:1 y 1:5, se correlaciona con los menores valores obtenidos para los parámetros $\mathrm{A}_{\max }$ $\mathrm{y} \mathrm{Cl}_{\text {tot }}$. De manera que, la aplicación de vinazas, en diferentes grados de dilución, afecta el contenido de nitrógeno foliar, el contenido de clorofilas totales y consecuentemente la asimilación fotosintética. Una tendencia similar se ha encontrado entre el contenido de nitrógeno foliar y la concentración de clorofila para girasol (Kumari, 2017). La disminución del contenido de $\mathrm{N}_{\mathrm{f}}$ por el agregado de vinazas, podría estar relacionada con el $\mathrm{pH}$ ácido de las mismas, lo cual afectaría la población de microorganismos del suelo relacionados con la mineralización del mismo y con ello la descomposición de la materia orgánica, nitrificación (Resende et al., 2006) y denitrificación (Leal et al., 1983).

Los resultados obtenidos a través de la medición de la relación $\mathrm{Fv} / \mathrm{Fm}$ pone de manifiesto la utilidad de la misma para detectar efectos a nivel del fotosistema II, ya que se trata de mediciones rápidas, no invasivas ni destructoras (Murchie \& Lawson, 2013). Estas mediciones pueden realizarse 


\section{J. A. González et al. - Vinazas y fotosíntesis en soja}

tanto a lo largo del ciclo de la planta como en ciertos estadios fenológicos, como se hizo en este estudio. Si bien diferentes tipos de estrés, como temperaturas extremas, falta de agua, fuerte intensidad lumínica, alta salinidad, falta de nutrientes, efecto de metales pesados, entre otros, afectan el funcionamiento del fotosistema II y que este efecto puede ser detectado a través de un cambio en la emisión de fluorescencia de la clorofila $a$, queda claro que ese cambio puede ser utilizado como una herramienta para conocer si el tratamiento que se analiza está impactando el fotosistema II, o bien para seleccionar variedades resistentes a determinadas condiciones de estrés. En el caso de cultivos, resulta un indicador temprano de estrés, de manera que se pueden tomar medidas de corrección antes que la planta llegue al estadio de adultez. En este estudio, el efecto del agregado de vinazas se puso de manifiesto como una disminución de la relación $\mathrm{Fv} /$ Fm, o sea un efecto directo sobre el fotosistema II, y como este efecto se recuperaba a medida que las diluciones iban disminuyendo. El efecto a nivel de $\mathrm{Fv} / \mathrm{Fm}$ y su recuperación a medida que la dilución de la vinaza disminuía, tuvo su correlato con los valores de $\mathrm{A}_{\max }$, CE y Tr medidos en los distintos tratamientos. Si bien algunos autores (Lambers et al., 2008) indican que un valor en la relación Fv/ Fm por debajo de 0,8 puede indicar condiciones estresantes en algunos casos, como en girasol, se han informado valores entre 0,7 y 0,85 y que bajo condiciones estresantes esos valores disminuían (Akram et al., 2012). En este estudio los valores de Fv/Fm en las plantas control oscilaron alrededor de 0,79 .

\section{Conclusiones}

Este estudio, más otras contribuciones sobre el mismo tema (González et al., 2014; González et al., 2018), demuestran que la aplicación de vinazas en bajas diluciones (1:1 y 1:5) afecta morfológicamente y fisiológicamente a las plantas de soja crecidas bajo condiciones de invernáculos. Estos efectos, se manifiestan en una reducción del contenido de nitrógeno foliar, el contenido de clorofilas totales y consecuentemente la asimilación fotosintética $\left(\mathrm{A}_{\text {net }}, \mathrm{A}_{\max }\right)$. También la TTE resulta afectada. A partir de la dilución 1:10 las variables mencionadas tienden a recuperarse.
Estos datos estarían indicando que el fertiriego con vinazas, para evitar problemas de estrés o de efectos a nivel del fotosistema II, sería posible utilizando diluciones mayores a 1:10. Aún así, si bien existe la posibilidad de usar vinazas, en la dilución señalada $u$ otras superiores, resulta necesario profundizar los estudios y realizar seguimientos o monitoreos de largo alcance (5 años o más) sobre los posibles efectos de la aplicación de vinaza en forma sostenida tanto sobre el rendimiento granario, potencial acumulación de metales pesados en los tejidos vegetales de las especies que se fertirrigen así como en las características fisicoquímicas y biológicas de los suelos. Los datos obtenidos muestran que independiente de la dilución utilizada, no se afecta la supervivencia de las plantas y las mismas llegan a cumplir con su ciclo. De manera que esto abre la posibilidad de utilizar la soja como un cultivo para la fitorremediación de los suelos enmendados con residuos de vinaza. Sin duda, esta posibilidad resulta ambientalmente interesante ya que se prevé que la destilación de alcohol y la consecuente producción de vinazas aumentará en los años próximos debido a la necesidad de nuevas fuentes de energía. Otro aspecto importante, que deberán incluirse en futuros estudios, es la generación de gases de efecto invernadero (GEI) que produce la vinaza como ya se demostró en otros países (Oliveira et al., 2013).

\section{Contribución DE LOS AUtORES}

JAG diseño, mediciones y escritura de manuscrito; FEP interpretación de datos, escritura de manuscrito; SB: mediciones y trabajo de laboratorio.

\section{Agradecimientos}

Al Ing. Haroldo Alemanny quién proveyó las semillas de soja para desarrollar este estudio. Este trabajo fue realizado con fondos de la Fundación Miguel Lillo (Miguel Lillo 251, T4000JFE, Tucumán, Argentina) dentro del proyecto B-00061. "Efecto de las vinazas sobre las primeras etapas de germinación y plántulas en especies nativas e introducidas". 


\section{Biblografía}

AKRAM, N.A., M. ASHRAF \& F.AL-QURANINY. 2012. Amino-levulinic acid-induced changes in some key physiological attributes and activities of antioxidant enzymes in sunflower (Helianthus annus L.) plants under saline regimes. Sci. Hortic. 142: 143-148.

ALVAREZ, S., P. EVELSON \& A. BOVERIS. 2008. Etanol: El combustible del futuro. Encrucijadas, 45: $1-7$.

BJÖRKMAN, O. 1968. Carboxydismutase activity in shade-adapted and sun-adapted species of higher plants. Physiol. Plant. 21: 1-10. https://doi.org/10.1046/j.0016-8025.2003.01067.x

BRACK, W. \& H. FRANK. 1998. Chlorophyll $a$ fluorescence: A tool for the investigation of toxic effects in the photosynthetic apparatus. Ecotoxicol. Environ. Saf. 40: 34-41. https://doi.org/10.1006/eesa.1997.1639

CHANFÓN CURBELO, J.M. \& LORENZO ACOSTA, Y. 2014. Alternativas de tratamiento de las vinazas de destilería. Experiencias nacionales e internacionales. Revista Centro Azúcar 41: 56-67.

CHRISTOFOLETTI, C.A., J.P. ESCHER, J.E. CORREIA, J.F. URBANO MARINHO \& C.S. FONTANETTI. 2013. Sugarcane vinasse: Environmental implications of its use. Waste Manag. 33: 2752-2761. https://doi.org/10.1016/j. wasman.2013.09.005

ESPAÑA-GAMBOA, E., J. MIJANGOS-CORTES, L. BARAHONA-PÉREZ, J. DOMÍNGUEZMALDONADO, G. HERNÁNDEZ-ZARATE \& L. ALZATE-GAVIRIA. 2011. Vinasses: characterization and treatments. Waste Manag. Res. 29: $1235-1250$.

https://doi.org/10.1177/0734242X10387313

EVANS, J.R. 1989. Photosynthesis and nitrogen relationships in leaves of $\mathrm{C} 3$ plants. Oecologia 78 : 9-19. https://doi.org/10.1007/BF00377192

FIELD, C. \& H. MOONEY. 1986. The photosynthesisnitrogen relationship in wild plants. In: GIVNISH, T.J. (ed.), On the Economy of Form and Function. pp. 25-55. Cambridge University Press, Cambridge, England.

GASTAL, F. \& G. LEMAIRE. 2002. N uptake and distribution in crops: An agronomical and ecophysiologica perspective. J. Exp. Bot. 53: 789799. https://doi.org/10.1093/jexbot/53.370.789

GONZÁLEZ, J.A, P. LANGUASCO \& F.E. PRADO. 2014. Efecto de las vinazas sobre la germinación de soja, trigo y quinoa en condiciones controladas. Bol. Soc. Argent. Bot. 49: 473-481.

GONZÁleZ, J.A., S.E. BUEDO, F.E. PRADO \& S. ÁLVAREZ. 2018. Efecto de la vinaza sobre el crecimiento y productividad de la soja (Glycine max) en condiciones semicontroladas. Bol. Soc. Arg. Bot. 53: 597-608. http://dx.doi.org/10.31055/1851.2372.v53.n4.21982

GONZÁLEZ MORENO, S., H. PERALES VELA \& M.O. SALCEDO ÁLVAREZ. 2008. La fluorescencia de la clorofila $a$ como herramienta en la investigación de efectos tóxicos en el aparato fotosintético de plantas y algas. $R A B$ 27: 119-129

KUMARI, S. 2017. Effects of nitrogen levels on anatomy, growth, and chlorophyll content in sunflower (Helianthus annuus L.) leaves. J. Agric. Sci. 9: 208219. https://doi.org/10.5539/jas.v9n8p208

LAMBERS, H., F.S. CHAPIN \& T.L. PONS. 2008. Plant Physiological Ecology. $2^{\circ}$ Edition. Springer-Verlag, New York, USA. https://doi.org/10.1007/978-0-387-78341-3

LARRAHONDO, J. 2009. La vinaza: Caracterización de la vinaza, usos y aprovechamientos potenciales. Memorias Seminario internacional de Fertilización y Nutrición de la Caña de Azúcar, Tecnicaña. Cali, Colombia, pp. 69-90.

LEDAL, J.R., N.M.B. AMARAL SOBRINHO, A.C.X. VELLOSO \& R.O.P. ROSSIELLO. 1983. Potencial redox e $\mathrm{pH}$ : variação em um solo tratado com vinhaça. Rev. Bras. Ciên. Solo. 7:257-261.

MARSCHNER, P. \& H. MARSCHNER. 2012. Marschner's Mineral Nutrition of Higher Plants. Academic Press, San Diego, CA, USA. https://doi.org/10.1016/C2009-0-63043-9

MILFORD, G.F.J., T.O. POCOCK \& J. RILEY. 1985. An analysis of leaf growth in sugar beet. I. Leaf appearance and expansion in relation to temperature under controlled conditions. Ann. Appl. Biol. 106: 163-172. https://doi.org/10.1111/j.1744-7348.1985.tb03106.x

MORANDINI, M. \& E. QUAIA. 2013. Alternativas para el aprovechamiento de la vinaza como subproducto de la actividad sucroalcoholera. Avance Agroind. 34: 1-17.

MUÑOZ, F. 2009. Resultados experimentales del uso de vinaza. Memorias Seminario internacional de Fertilización y Nutrición de la Caña de Azúcar, Tecnicaña. Cali, Colombia, pp. 19-20.

MURCHIE, E.H. \& T. LAWSON. 2013. Chlorophyll fluorescence analysis:a guide to good practice and understanding some new applications. J. Exp. Bot. 64:3963-3998. https://doi.org/10.1093/jxb/ert208

OLIVEIRA, B.G., J.L.N. CARVALHO, C.E.P. CERRI, C.C. CERRI \& B.J. FEIGL. 2013. Soil greenhouse gas fluxes from vinasse application in Brazilian sugarcane areas. Geoderma 200/201: 77-84. http://dx.doi.org/10.1016/j.geoderma.2013.02.005

PÉREZ-BERMÚDEZ, I. \& N. GARRIDO-CARRALERO. 2006. Aprovechamiento integral de vinazas de destilerías. Una revisión actual. Revista Ingeniería Química 38: 129-133. 


\section{J. A. González et al. - Vinazas y fotosíntesis en soja}

RAWSON, H.M., J.E. BEGG \& R.G. WOODWARD. 1977. The effect of atmospheric humidity on photosynthesis, transpiration and water use efficiency of leaves of several plant species. Planta 134: 5-10.

RESENDE DE, A.S., R.P. XAVIER, O.C. DE OLIVEIRA, S. URQUIAGA, B.J.R. ALVES \& R.M. BODDEY. 2006. Long-term effects of pre-harvest burning and nitrogen and vinasse applications on yield of sugar cane and soil carbon and nitrogen stocks on a plantation in Pernambuco, N.E. Brazil. Plant and Soil 281: 339-351. https://doi.org/10.1007/s11104-005-4640-y

ROBERTIELLO, A. 1982. Upgrading of agricultural and agroindustrail wastes: the treatment of destillery effluents (vinasses) in Italy. Agric. Wastes 4: 387395. https://doi.org/10.1016/0141-4607(82)90033-6

SALAZAR-PARRA, C., J. AGUIRREOLEA, M. SANCHEZ-DIAZ, J.J. IRIGOYEN \& F. MORALES. 2012. Climate change (elevated $\mathrm{CO}_{2}$, elevated temperature and moderate drought) triggers the antioxidant enzymes' response of grapevine cv. Tempranillo, avoiding oxidative damage. Physiol. Plant. 144: 99-110. https://doi.org/10.1111/j.1399-3054.2011.01524.x

SANCHEZ MOORE, C.C., A. RODRIGUES NOGUEIRA \& L. KULAY. 2017. Environmental and energy assessment of the substitution of chemical fertilizers for industrial wastes of ethanol production in sugarcane cultivation in Brazil. Int. J. Life Cycle Assess. 22: 628-643.

https://doi.org/10.1007/s11367-016-1074-0

SCHREIBER, U., W. BILGER, H. HORMANN \& C. NEUBAUER. 2000. Chlorophyll fluorescence as a diagnostic tool: Basics and some aspects of practical relevance. In: RAGHAVENDRA, A.S. (ed.), Photosynthesis. Comprehensive Treatise. pp. 320-336. Cambridge University Press, Cambridge, England.

SCHULTE, M., C. OFFER \& U. HANSEN. 2003. Induction of $\mathrm{CO}_{2}$-gas exchange and electron transport: comparison of dynamic and steady-state responses in Fagus sylvatica leaves. Trees 17: 153163.

https://doi.org/10.1007/s00468-002-0219-x

SKILLMAN, J.B. 2008. Quantum yield variation across the three pathways of photosynthesis not yet out of the dark. J. Exp. Bot. 59: 1647-1661. https://doi.org/10.1093/jxb/ern029

TRÁPANI, N. \& A.J HALL. 1996. Effects of level of insertion and nitrogen supply on the expansion of leaves of field-grown sunflower (Helianthus annus L.). Plant Soil 184: 331-340. https://doi.org/10.1007/BF00010462

VOS, J. \& H. BIEMOND. 1992. Effect of nitrogen on the development and growth of the potato plant. I. leaf appearances, expansion growth, life span of leaves and stem branching. Ann. Bot. 70: 27-35. 
